\title{
ArcheoSciences
}

Revue d'archéométrie

$34 \mid 2010$

Varia

\section{Une pollution métallique antique en haute vallée de l'Arve}

An antique metallic pollution in high valley of Arve river

Fabien Arnaud, Joël Serralongue, Thierry Winiarski et Marc Desmet

\section{OpenEdition}

1 Journals

Édition électronique

URL : https://journals.openedition.org/archeosciences/2759

DOI : $10.4000 /$ archeosciences. 2759

ISSN : 2104-3728

Éditeur

Presses universitaires de Rennes

Édition imprimée

Date de publication : 10 avril 2010

Pagination : 197-201

ISBN : 978-2-7535-1407-2

ISSN : 1960-1360

Référence électronique

Fabien Arnaud, Joël Serralongue, Thierry Winiarski et Marc Desmet, « Une pollution métallique antique en haute vallée de l'Arve », ArcheoSciences [En ligne], 34 | 2010, mis en ligne le 11 avril 2013, consulté le 03 février 2022. URL : http://journals.openedition.org/archeosciences/2759 ; DOI : https://doi.org/ 10.4000/archeosciences. 2759

Article L.111-1 du Code de la propriété intellectuelle. 


\title{
Une pollution métallique antique en haute vallée de l'Arve
}

\section{An antique metallic pollution in high valley of Arve river}

\author{
Fabien Arnaud*, Joël Serralongue**, \\ Thierry Winiarski ${ }^{* * *}$ et Marc DeSMet ${ }^{* * * *}$
}

\begin{abstract}
Résumé : Une séquence sédimentaire de huit mètres, couvrant les derniers 2000 ans a été prélevée dans le lac d'Anterne (Haute-Savoie, France) par $13 \mathrm{~m}$ de fond. Après datation par le radiocarbone une série d'analyses des concentrations en plomb a été réalisée suivant une résolution d'environ 40 ans. Cette série met en évidence une pollution importante par ce métal au cours du $\mathrm{II}^{\mathrm{e}}$ siècle de notre ère. Si les traces archéologiques d'exploitation minière antique demeurent à ce jour introuvables dans la région, l'intensité même de la pollution ne laisse guère de doute sur une origine proximale. Nos données remettent en cause une nouvelle fois l'idée d'une origine distante de la contamination généralisée de l'Europe à la période antique, en provenance notamment de la mine espagnole de Rio Tinto. Elles ouvrent tout un champ de recherche à la croisée des géosciences et des sciences archéologique, voire historiques, pour tenter d'identifier les centres locaux de production et/ou de traitement de minerai de plomb argentifere dans les Alpes et les autres districts miniers européens.
\end{abstract}

\begin{abstract}
A 8m-long sediment core, covering the last 2000 years was taken in Lake Anterne (Haute-Savoie, France) under $13 \mathrm{~m}$ water depth and radiocarbon dated. A 40 years average resolution series of lead concentration was subsequently acquired. This series shows a prominent lead contamination dates $2^{\text {nd }}$ century $A D$. Whereas archaological evidences of mining exploitation remain unfoundable in the region, the intensity of the contaminaiton peak argues for a local origin of it. Our data one more time lead to relativise the theory according to which antique lead contamination uniquely originate form the exploitation of the Spanish Rio Tinto mine. Such a study open a wide research field, crossing geo- and archaeological sciences, attempting to identify local centers of production and transformaiton of lead-silver ore in the Alps and other mining districts.
\end{abstract}

Mot clé : Contamination métallique, Antiquité, Plomb, Sédiment, Alpes.

Key words: Metal contamination, Antiquity, Lead, Sediment, Alps.

\section{INTRODUCTION}

L'étude des concentrations et signatures isotopiques des polluants métalliques dans les glaces du Groenland a permis de démontrer que l'impact de l'Homme sur les écosystèmes distants était mesurable dès la période romaine (Hong et al., 1994). S’il a été montré qu'une grande part de la pollution antique au Groenland provenait de la mine espagnole de Rio Tinto (Rosman et al., 1997), la question de la provenance de la pollution en Europe reste posée.

* EDYTEM, Université de Savoie, CNRS - Pôle montagne, F-73373 Le Bourget-du-Lac.

** Service archéologique, Conseil Général de la Haute-Savoie - BP 2444, F-74041 Annecy Cedex.

*** Laboratoire des Sciences de l'Environnement, ENTPE - F-69518 Vaulx-en-Velin.

**** ISTO, Université François-Rabelais Tours, CNRS, GéEAC - Faculté des Sciences \& Tech. Parc Grandmont 37200 Tours. 
F. Monna (Monna et al., 2004b) a récemment exposé les bases d'une approche résolument pluridisciplinaire de l'histoire des pollutions anciennes démontrant l'intérêt scientifique d'un travail concerté entre géochimistes et archéologues. Cette approche est actuellement mise en œuvre dans le Massif Central (Baron et al., 2005, Monna et al., 2004b) et les Pyrénnées (Monna et al., 2004a), mais les Alpes n'ont été que peu étudiées et les rares études sur la contamination au plomb sont restées cantonnées aux derniers siècles, que ce soit à partir d'archives glaciaires (Rosman et al., 2000) ou lacustres (Arnaud et al., 2004). Au moins en ce qui concerne les Alpes du Nord, ce relatif désintérêt est probablement dû à la rareté de traces attestées d'exploitations anciennes. A l'époque romaine les seules exploitations connues de plomb argentifère sont celles de Tarentaise mais d'autres sont suspectées dans la haute vallée de l'Arve. C'est dans cette région que nous avons réalisé un sondage lacustre dans le but de poser un premier jalon dans la reconstitution de l'histoire de la contamination antique au plomb dans les Alpes Françaises du Nord (fig. 1). Une étude précédente (Arnaud et al., 2004) avait montré la qualité d'enregistreur de la contamination atmosphérique de ce lac d'altitude $(2061 \mathrm{~m})$. En outre, la sédimentation varvée du Lac d'Anterne a permis d'établir un modèle d'âge précis et ainsi de confronter une série de concentration en plomb à l'histoire économique supposée de la région de Passy durant les tous premiers siècles de notre ère.

\section{RÉSUltats/DisCUSSION}

Nous avons identifié dans les sédiments du lac d'Anterne un pic majeur de concentration en plomb au moins antérieur à 120 apr. J.-C. (âge du plus vieil échantillon mesuré) qui s'étale jusque vers 300 apr. J.-C (fig. 2). A cette époque, la région est occupée par les Romains qui ont conquis le territoire des Allobroges en 121 av. J.-C. Le maximum de contamination (220 apr. J.-C.) correspond par ailleurs à l'apogée de la civilisation romaine dans les Alpes (Bertrandy, 2000).

Or les seules exploitations de plomb connues dans la région se situent en Tarentaise, à proximité des villages actuels de Macôt et de Pesey, celles de la haute vallée de l'Arve n'étant encore qu'hypothétiques. Celles-ci pourraient se situer aux environs de Passy (Bertrandy, 2000), où plusieurs témoins archéologiques accréditent l'hypothèse de l'existence toute proche de richesses minières considérables ou jugées comme telles à l'époque (Bertrandy et al., 1999, p. 283-288).

Le premier indice a été observé en rive gauche de l'Arve face au village de Servoz (fig. 3) : il s'agit d'une galerie souterraine qui a donné son nom au seuil rocheux qu'elle traverse, La Rateriaz (ratière ou galerie); d'une longueur originelle estimée entre 65 et $70 \mathrm{~m}$ pour une hauteur de 1,70 à $2,00 \mathrm{~m}$ et une largeur de $0,90 \mathrm{~m}$, elle fut découverte et détruite sur une vingtaine de mètres en 1963 lors du percement du tunnel routier, emprunté de nos jours par la voie descendante menant de Chamonix à Sallanches. Les investigations les plus récentes, menées en 1994 lors de l'élargissement de cet
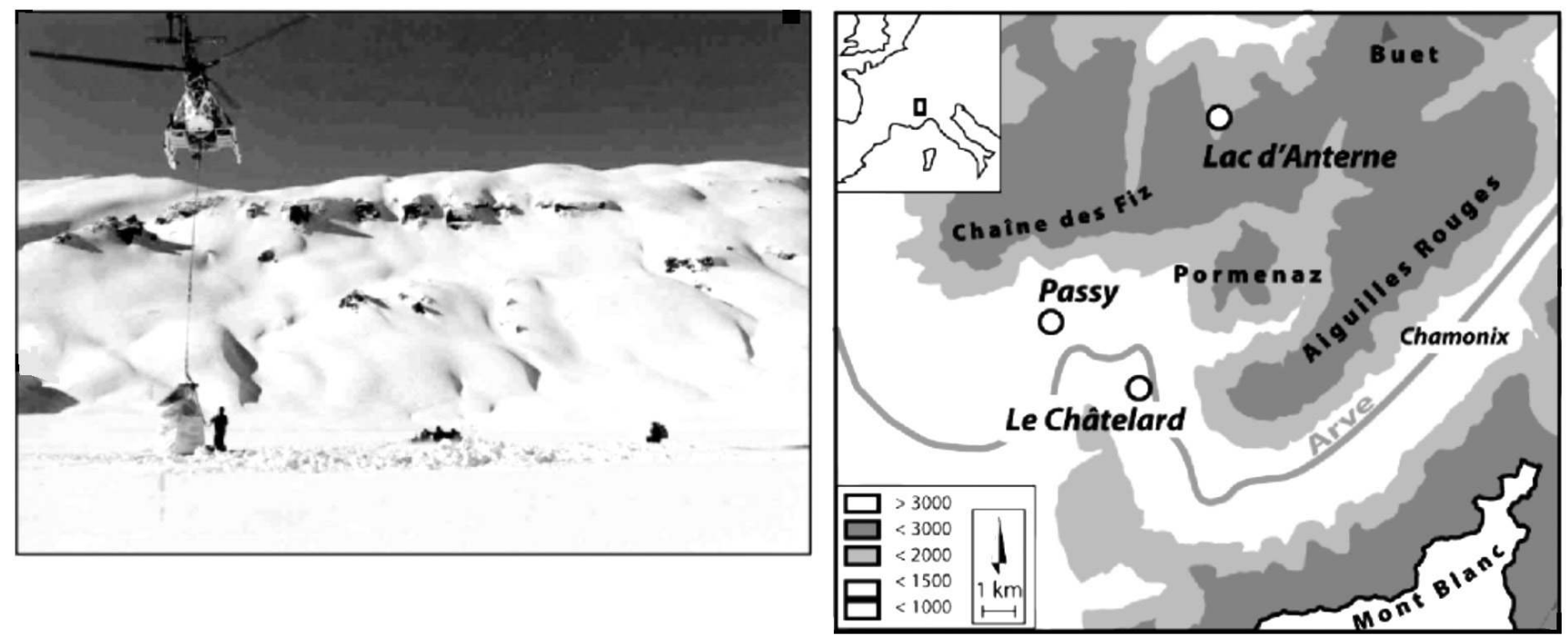

Figure 1 : Pose du carottier sur le lac d'Anterne et carte du secteur à l'étude dans la haute vallée de l'Arve.

Figure 1: Location map of the studied lake and deposit osf coring material on the ice-covered Lake Anterne. 


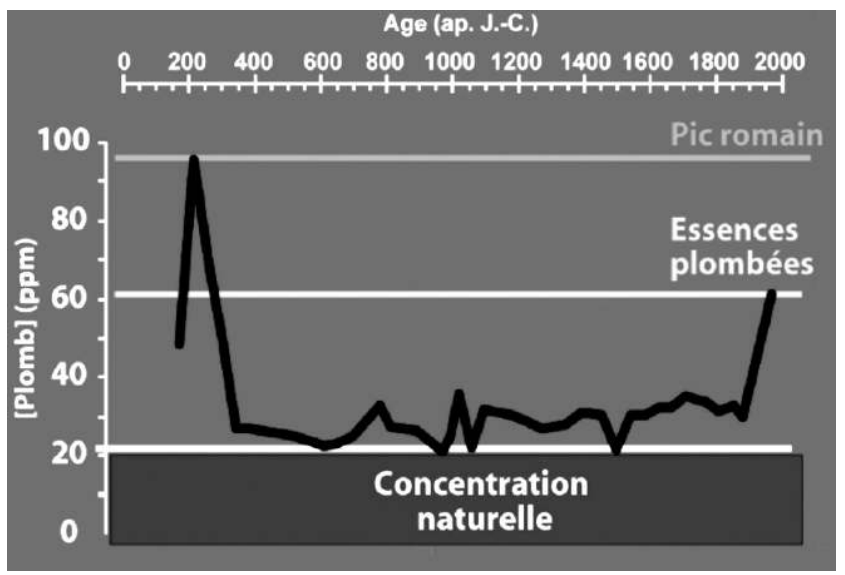

Figure 2 : La série de plomb du lac d'Anterne couvrant les derniers 1800 ans.

Figure 2: The lead concentration series from Lake Anterne covering the last 1800 years.

ouvrage d'art, ont confirmé son rôle d'aqueduc, pouvant être transformé en réservoir en fermant des vannes situées à chacune de ses extrémités (Cécillon, 1994).

Cette " chasse hydraulique ", de par les travaux considérables qu'a entraîné son percement dans le roc, ne peut trouver sa raison que dans les installations métallurgiques repérées au XIX ${ }^{\mathrm{e}}$ siècle, dans le vallon du Châtelard sur lequel elle débouche. D'une contenance proche de $120 \mathrm{~m}^{3}$, elle était alimentée en amont par un bief, dont il reste à déterminer l'approvisionnement - Arve ou ruisseaux descendant du Prarion -; en aval du vallon du Châtelard, elle empruntait un canal à ciel ouvert, également taillé dans le roc, encore bien visible de nos jours au lieudit Les Egratz (de la configuration en escaliers de l'ancienne route), où taillé en degrés, il se précipite vers l'Arve telle une conduite forcée. L'analyse de l'architecture et des modes opératoires utilisés pour construire ces installations ont permis de les dater de l'époque romaine (Cécillon, 1994) mais rien ne permet d'affiner cette datation ni de préciser la teneur des minerais exploités.

Le deuxième indice a son origine en rive droite de l'Arve, dans l'enceinte d'un temple romain, implanté à quelques centaines de mètres du vieux village de Passy, face au massif du mont Blanc (fig. 4).

Là, trois inscriptions lapidaires, aujourd'hui conservées sous le porche de l'église paroissiale, témoignent d'un culte rendu au dieu Mars par des personnages importants de la cité de Vienne. De fait, il s'agit de trois magistrats, ayant eu successivement les fonctions les plus élevées de l'administration municipale viennoise : le premier était triumuir chargé de l'entretien et de la surveillance des lieux publics,

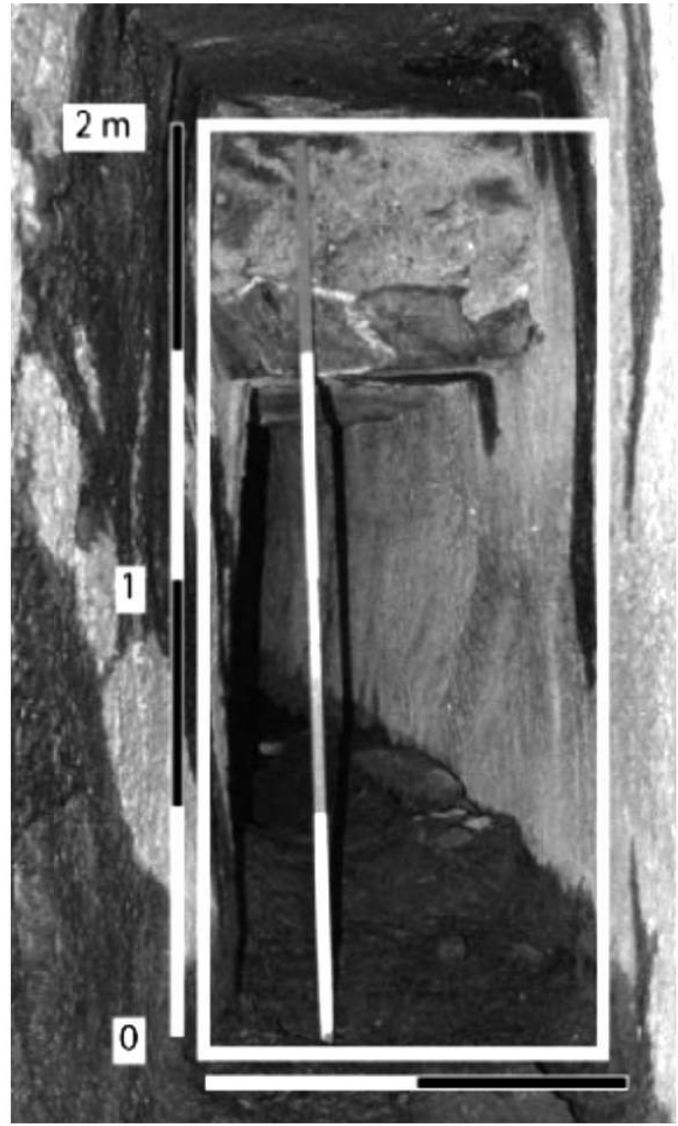

Figure 3 : (Voir planche couleur) Détail de la galerie romaine de La Ratériaz, supposée être une chasse hydraulique destinée à une probable exploitation métallurgique située dans le vallon du Châtelard; cl. J. Serralongue, 1994.

Figure 3: (See colour plate) Detail of the so-called La Ratériaz Roman gallery, supposed to have been used for hydraulic purposes in relation with a metallurgical plant downstream the so-called Vallon $d u$ Châtelard. Photo by J. Serralongue (1994).

le deuxième était questeur des deniers publics et duumuir du trésor, fonction occupée également par le troisième (Rémy, 1995). Même si l'on peut admettre que le dieu Mars, adoré à Passy en tant que dieu guérisseur comme l'atteste l'une des dédicaces (fig. 1), avait une réputation considérable, on peut s'étonner de la présence en ces lieux si éloignés de Vienne d'une telle densité de notables. Ils appartiennent aux trois principaux collèges de magistrats, créés en Viennoise dans les années 40-50 apr. J.-C., quand la cité fut promue au rang de « colonie romaine honoraire », et l'un d'entre eux occupe le sommet de la hiérarchie.

Dès lors, il est tentant de lier la présence de ces hauts personnages de la cité, en charge des finances et de la gestion des domaines publics, aux probables contrôles qu'ils étaient en droit d'effectuer dans ces montagnes - propriétés de l'État 


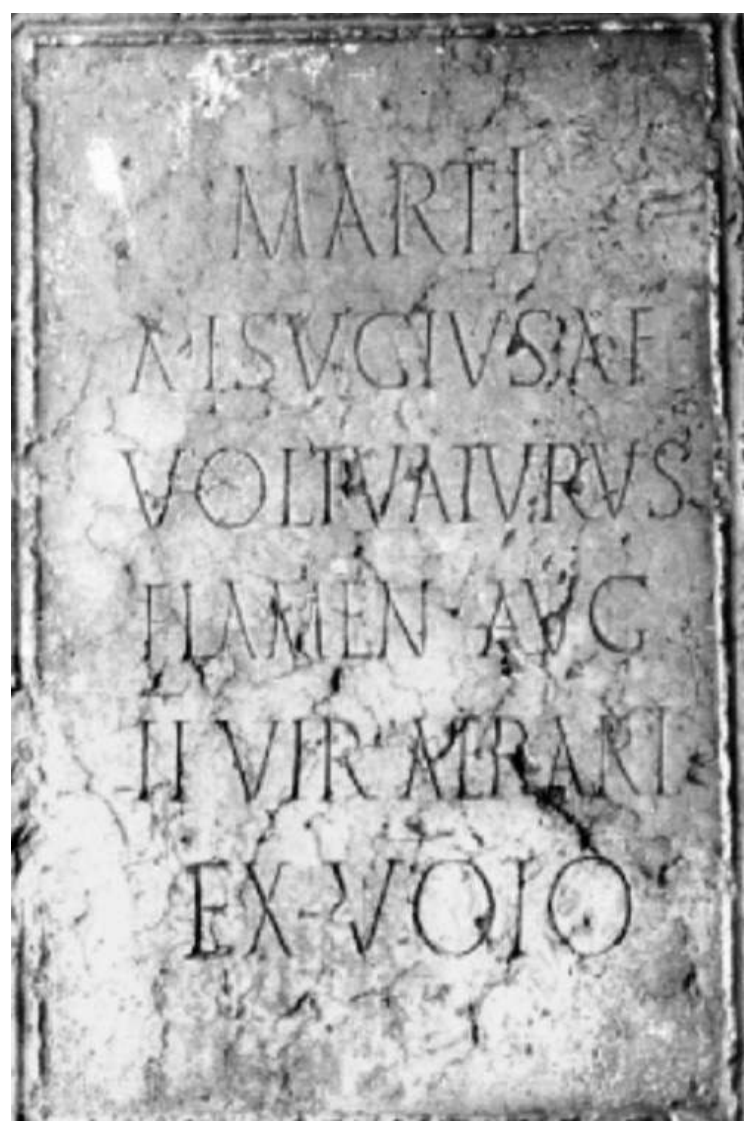

Figure 4 : Ex-voto en calcaire $(90$ x $60 \mathrm{~cm})$, retrouvé dans le temple de Passy, dédié au dieu Mars : "A Mars, Aulus Isugius Vaturus, de la tribu Voltinia, flamine impérial, duumvir du trésor, à la suite d'un vœu ". CIL XII 2349; cl. J. Serralongue.

Figure 4: Limestone ex-voto $(90 \times 60 \mathrm{~cm})$, found in a temple dedicated to god Mars in the village of Passy: "To mars, Aulus Isugius Vaturus, from the Voltina tribe, imperial flamine, treasure duumvir, following a wish". CIL XII 2349. Photo by J. Serralongue.

ou de la cité - et tout particulièrement des méthodes mises en ouvre pour en exploiter et en transformer les richesses minières (Serralongue, 1995).

Vienne, chef-lieu de la cité, rassemblait le plus grand nombre d'ateliers de plombier recensés en Gaule - 70 sont attestés - et leurs exigences en matière première étaient sans doute l'objet de fructueux marchés. Abondamment utilisé dans les travaux hydrauliques (tuyaux d'adduction d'eau, réservoirs et chaudières), mais également dans la construction (scellement des blocs, couverture de certains bâtiments), ou dans la marine (ancre), le plomb est aussi destiné à la fabrication d'objets votifs offerts aux dieux (rouelles, objets miniatures), et dans le domaine militaire à la fabrication de balles de fronde ou encore dans le domaine du funéraire à celle d'urnes cinéraires et de cercueils (Cochet,
2000; Savay-Guerraz, 2001). Il est par ailleurs notable que le plomb est également un produit dérivé de l'exploitation de l'argent, métal d'une grande importance dans la société romaine qui battait monnaie en argent. L'étymologie de quelques lieux-dits atteste de l'exploitation passée d'argent dans les montagnes environnant Passy. Quoiqu'il en soit, il est possible que le besoin en matière première, que ce soit en plomb ou en argent, se soit traduit par une volonté politique d'en organiser l'extraction ce qui expliquerait l'établissement d'un complexe de collecte et de transformation de minerai à Passy. Par son intensité, la contamination romaine du Lac d'Anterne semble confirmer l'idée de l'existence d'un tel centre dans la haute vallée de l'Arve.

Ces pistes étant posées, il est toutefois évident que la preuve définitive de l'existence d'exploitations minières et/ ou d'activités métallurgiques de grande ampleur en HauteSavoie ne pourra être apportée que des indices archéologiques directs. Force est de constater qu'hélas, aujourd'hui tel n'est pas le cas et notre hypothèse, pour cohérente qu'elle apparaisse ne peut être aujourd'hui considérée comme absolument valide.

\section{Conclusion}

Si une grande partie du plomb extrait durant l'Antiquité provient de la mine espagnole de Rio Tinto (Rosman et al., 1997), les résultats acquis au Lac d'Anterne attestent de l'existence d'exploitations secondaires ayant eu un impact environnemental parfois très important. Les modalités de la contamination de l'environnement par le plomb à l'époque romaine pourraient ainsi être plus complexes que ne le laissent penser les seules données acquises au Groenland, tout au moins au cœur de l'Empire. Une étude spatiale de la contamination métallique à cette époque pourrait ainsi servir de modèle à la compréhension des modes de dispersion de tels contaminants.

De nouvelles recherches autour du Massif du mont Blanc permettront de préciser la provenance de la contamination en couplant une étude spatiale de la contamination dans différentes archives naturelles et la caractérisation isotopique du contaminant à la fois dans les archives naturelles, dans les filons potentiels et dans les objets archéologiques supposés produits à partir du plomb de Passy. Nous nous attacherons également à caractériser l'éventuel impact écologique de la péruiode d'intense exploitation romaine au travers d'une étude paléoécologique (pollens, chironomes, charbons). Cette approche basée sur l'étude d'archives naturelles sera menée en parallèle d'investigations archéologiques visant à répertorier sur le terrain les excavations ayant pu être exploitées à la période romaine. 


\section{Bibliographie}

Arnaud, F., Revel-Rolland, M., Bosch, D., Winiarski, T., Chapron, E., Desmet, M., Tribovillard, N. et Givelet, N., 2000. A 300 years-long history of lead contamination in Northern French Alps from distant lake sediment records, Journal of Environmental Monitoring, 6, p. 448-456.

Baron, S., Lavoie, M., Ploquin, A., Carignan, J., Pulido, M. et Beaulieu, J.-L. de, 2005. Record of metal workshops in peat deposits: History and environmental impact on the Mont Lozère Massif, France, Env. Sci. and Tech., 39, p. 5131-5140.

Bertrandy, F., 2000. La Savoie à l'époque romaine, [http://www. sabaudia.org/v2/dossiers/savoieromaine/public1.php].

Bertrandy, F., Chevrier, M. et Serralongue, J., 1999. La Haute-Savoie (74), coll. "Carte Archéologique de la Gaule ", Académie des Inscriptions et Belles Lettres, Paris, 412 p.

Cécillon, C., 1994. Passy, le Châtelard in Chronique des découvertes archéologiques dans le département de la Haute-Savoie, Revue Savoisienne, 134 : 47-50.

Cochet, A., 2000. Le plomb en Gaule romaine, techniques de fabrication et produits, Monographies Instrumentum 13, Montagnac, éd. M. Mergoil, 223 p.

Hong, S., Candelone, J.-P., Patterson, C. C., Boutron, C. F., 1994. Greenland ice evidence of hemispheric lead pollution two millennia ago by Greek and Roman civilizations, Science, 265, p. 1841-1843.
Monna, F., Galop, D., Carozza, L., Tual, M., Beyrie, A., Marembert, F., Chateau, C., Dominik, J. et Grousset, F. E., 2004a. Environmental impact of early Basque mining and smelting recorded in a high ash minerogenic peat deposit. Sc. of The Total Environ., 327, p. 197-214.

Monna, F., Petit, C., Guillaumet, J.-P., Jouffroy-Bapicot, I., Blanchot, C., Dominik, J., Losno R., Richard H., LévêQue J. et Chateau C., 2004b. History and Environmental Impact of Mining Activity in Celtic Aeduan Territory Recorded in a Peat Bog (Morvan, France), Environ. Sci. and Tech., 38, p. 665673.

RémY, B. (dir.), 1995. Inscriptions Latines de Haute-Savoie, Annecy, Musée-Château.

Rosman, K. J. R., Chisholm, W., Hong, S., Candelone, J. P. et Boutron, C. F., 1997. Lead from Carthaginian and Roman Spanish mines isotopically identified in Greenland ice dated from $600 \mathrm{BC}$ to $300 \mathrm{AD}$, Environ. Sci. and Tech. 31, p. 34133416.

Rosman, K. J. R., Ly, C., Van de Velde, K. C. et Boutron, F., 2000. A two century record of lead isotopes in high altitude Alpine snow and ice, Earth. Planet. Sci. Lett., 176, p. 413-424.

Savay-Guerraz, H., 2000. Plomb, plomb, plomb, plomb..., Exposition-actualité réalisée par le département du Rhône, $16 \mathrm{p}$.

Serralongue, J., 1995. Passy in Rémy B. (dir.), Inscriptions Latines de Haute-Savoie, Annecy, Musée-Château, p. 20. 
